Jens van Scherpenberg

\title{
Finanzkapital, Finanzkrise und internationale Staatenkonkurrenz
}

\begin{abstract}
Um die Auswirkungen der Finanzkrise von 2007-2010 auf die internationalen Beziehungen zu erfassen, ist es unerlässlich, die wesentlichen wirtschaftlichen Triebkräfte $z u$ verstehen, die der Krise zu Grunde liegen und die auch entscheidend die nationalen Reaktionen auf die Krise bestimmen. Hierzu werden, aufbauend auf Marx' KapitalAnalyse, zunächst drei Thesen zur »Systemrelevanz " des Finanzkapitals vorgestellt. Mit der darin aufgezeigten grundlegenden und umfassenden Subsumtion aller Bereiche der kapitalistischen Wirtschaft unter den selbstreferentiellen Akkumulationsprozess des Finanzkapitals wird zugleich die Finanzialisierungsthese als Erklärungsansatz kritisiert. Aus der Orientierung der Staaten an der Sicherung optimaler Akkumulationsbedingungen für das von ihrem Standort aus operierende Finanzkapital lässt sich ableiten, warum es etwa im G20-Rahmen zu keinen wirklich substantiellen Vereinbarungen globaler Finanzmarktregulierung, sondern vielmehr zu einer Intensivierung der Konkurrenz um die Gestaltung der künftigen internationalen Wirtschafts- und Finanzordnung kommt. Am dialektischen Verhältnis von Rivalität und Interdependenz in den Wirtschafts- und Währungsbeziehungen zwischen den USA und China wird diese Aussage erläutert.
\end{abstract}

\section{Einleitung}

Unzweifelhaft wirft die gegenwärtige globale Finanzkrise mit ihren absehbaren Verwerfungen im bisherigen Kräfteverhältnis der Nationen wie in den Strukturen und Funktionen internationaler Institutionen und mit ihren Herausforderungen für Global Governance eine Fülle neuer, herausfordernder Forschungsfragen für die Internationale Politische Ökonomie auf. Nur wenige dieser Fragen lassen sich jedoch sinnvoll klären, wenn die Finanzkrise als quasi exogener Schock für die internationale Ordnung betrachtet wird. Umso wichtiger sind präzise Beiträge zur Genese der Krise und ihren politischen Hintergründen in einem IB-/IPÖ-bezogenen Kontext, wie sie etwa Hans Jürgen Bieling (2009) und Brigitte Young (2009) bieten. Dies um so mehr, als die Wirtschaftswissenschaft im engeren Sinne, also diejenige akademische Disziplin, deren Denkansätze, Methoden und Schlussfolgerungen vielleicht am stärksten durch die Krise diskreditiert worden sind, wie unter anderem Paul Krugman (2009) zeigt, sich auch bei deren Ex-Post-Erklärung nachhaltig schwer tut. Gleichwohl bleiben auch die aus der Internationalen Politischen Ökonomie kommenden bisherigen Erklärungsansätze unbefriedigend, so weit es darum geht, den endogenen, im Finanzsektor selbst zu suchenden Grund für die erheblichen Probleme abgestimmter internationaler Regulierung der internationalen Finanzmärkte zu ermitteln, wie sie auf den 
letzten beiden Gipfelkonferenzen der Gruppe der 20 in London (April 2009) und Pittsburgh (September 2009) deutlich wurden.

Im ersten Teil der folgenden Ausführungen werden daher jenseits unmittelbarer mehr oder weniger zufälliger - Krisen-Anstöße einige kurze, thesenhafte Aussagen zu den Gründen der Finanzkrise formuliert, aus denen im zweiten Teil versucht wird, die Ursachen für das Versagen nationaler und internationaler Finanzmarktregulierung in der Krisenprävention abzuleiten. Ein abschließender dritter Teil wendet die gewonnenen Aussagen zur internationalen Regulierungskonkurrenz auf die Verhandlungen im Rahmen von G-20 und IWF an. In meinen Thesen setze ich mich nicht nur mit den Forums-Beiträgen zur Finanzkrise aus Heft 1/2009 der ZIB, sondern auch mit der derzeitigen, durchaus kontroversen »innermarxistischen« Diskussion in Deutschland zu diesem Thema (z. B. Altvater 2009) kritisch auseinander, beziehe mich dabei jedoch selbst auf Marx, dessen Kapital-Analyse auch für das Verständnis der Reaktion der in der Gruppe der 20 zusammengeschlossenen führenden Wirtschaftsmächte auf die Krise nützlich ist.

\section{Die Akkumulation des Finanzkapitals}

Im Zug der Finanzkrise ist die Kritik der politischen Ökonomie des Kapitalismus, die Karl Marx in seinem dreibändigen Hauptwerk leistete, zwar wieder aus dem Müllhaufen der Geschichte ausgegraben worden, auf den sie nach 1990 wohl etwas voreilig geworfen wurde. Fehlperzeptionen der Marxschen Analyse bleiben jedoch auch unter denen verbreitet, die sich auf ihn berufen. Marx hat nicht die Krise des Kapitalismus vorhergesagt, die viele heutige Kapitalismuskritiker - beispielhaft sei hier Elmar Altvater (2009) genannt - ausrufen, schon allein deswegen nicht, weil er sich nicht als Vorhersager der Zukunft verstand, vielmehr lediglich aus der Logik des historischen Prozesses, wie er ihn analysierte, resultierende Widersprüche und Möglichkeiten zu deren Überwindung aufzeigen wollte. In diesem Sinne hat er analysiert, dass Krisen notwendige Begleiterscheinung des Kapitalismus sind und wie sie sich notwendig als Kreditkrisen, also Finanzkrisen entfalten. Und er hat klar gesagt, dass jede Krise zugleich wieder die Grundlage für einen neuen Akkumulationszyklus schafft.

Mit den folgenden drei Thesen, die auf Marx' Analyse des »zinstragenden « und »Bankkapitals« (Marx 2003) aufbauen, möchte ich aufzeigen, wie sich die »Systemrelevanz« des Finanzsektors in den entwickelten kapitalistischen Staaten aus den Besonderheiten der Akkumulation des Finanzkapitals ergibt und welche Folgen dies für die Möglichkeiten und Grenzen internationaler Kooperation und Koordination bei der Finanzmarktregulierung hat.

(1) Die Logik der Akkumulation des Finanzkapitals besteht darin, Kredit, also Schulden in Kapital zu verwandeln, indem die Finanzinstitute ihr Aktivgeschäft laufend refinanzieren und so ihr angewandtes Kapital weit über die Grenzen ihres Eigenkapitals hinaus erhöhen. Das Generieren und Kapitalisieren von Schulden also, finanztechnisch auch leveraging genannt, ist die Quelle der Akkumulation des Finanzkapitals, mit der es sich von der »realen« Akkumulation über den kapitalistischen 
Produktionsprozess emanzipiert und »als Kapitalverhältnis seine äußerlichste und fetischartigste Form (erreicht)«(Marx 2003: 404). Das Gelingen dieser Akkumulation steht unmittelbar unter der einzigen, allerdings zwingenden Bedingung, dass das als Schuldner fungierende Finanzinstitut jederzeit den Beweis seiner Liquidität antreten kann. Solange es diesen Beweis antreten kann, wird er in der Regel nicht gefordert, sondern durch Vertrauen in seine Liquidität ersetzt. Insoweit ist seiner Kapitalakkumulation prinzipiell keine Schranke gesetzt. Wird der Liquiditätsbeweis doch einmal am Markt eingefordert, kommt das meist bereits einem Misstrauensvotum gleich und stürzt das betreffende Kreditinstitut daher in der Regel sogleich in eine existenzbedrohende Krise, da dann die normalen Quellen seiner Liquiditätsbeschaffung versiegen. Ist diese Illiquidität nicht nur auf ein Misstrauensvotum gegenüber der Kreditwürdigkeit einer einzelnen Bank zurückzuführen, sondern auf den als unsicher angesehenen Kapitalrückfluss aus einer ganzen Kategorie von Schuldpapieren, setzt die Illiquidität der ersten Bank eine Kettenreaktion in Gang, da sie die Nachfrage nach Liquiditätsbeweisen, also den Zweifel an der Liquidität des Finanzsektors insgesamt sprunghaft steigen lässt: aus der Illiquidität einer Bank wird eine allgemeine Finanzkrise mit massiver Kapitalentwertung und daraus folgender verbreiteter Bankeninsolvenz. Dass bei dieser Krise im Subprime-Sektor engagierte Banken die ersten waren, die insolvent wurden, ist zwar im Nachhinein einleuchtend, aber keine logische Notwendigkeit. Es gab hinreichend andere Schuldenkategorien, die die Kettenreaktion der Illiquidität und Insolvenz hätten in Gang setzen können, wie Kommunalobligationen, Verbraucherkredite, Kredite an Private Equity Funds zur Finanzierung von Unternehmenskäufen, ganz zu schweigen von dem gigantischen Volumen der außerbörslich (over-the-counter: OTC) gehandelten Derivate, die Ende Dezember 2008 ein Volumen von 592 Billionen US-Dollar zugrunde liegender Basiswerte und einen Marktwert von 34 Billionen Dollar erreichten, gegenüber einem Welt-Bruttoinlandsprodukt von 61 Billionen Dollar. ${ }^{1}$ Der Ersatz der wegen der massiven Entwertungen von als Kapital fungierenden Schuldtiteln im Finanzsektor abhanden gekommenen Liquidität durch vom Staat bereitgestellte Liquidität ist nicht mehr als eben dies: Ersatz für ausbleibenden Rückfluss aus Aktiva der Banken und insofern Abwendung von Insolvenz. Ausweis funktionierender Kapitalakkumulation ist er jedenfalls nicht.

(2) Aus der Logik seiner Akkumulation heraus, wie sie sich in der Konkurrenz der Finanzinstitute durchsetzt, überwindet das Finanzkapital die Schranken der primären Schuldner-Gläubiger-Beziehung zu einem eigenständigen, selbstreferentiellen Akkumulationsprozess »fiktiven Kapitals«, wie Marx es nennt. ${ }^{2}$ Es entwickelt immer

1 OTC-Daten: Bank für Internationalen Zahlungsausgleich, (http://www.bis.org/statistics/derstats.htm; 5.10.2009), Welt-BIP: CIA World Factbook (Schätzung) (https://www.cia.gov/ library/publications/the-world-factbook/geos/xx.html; 5.10.09.

2 Obwohl Marx zu Beginn des 25. Kapitels des Kapital, Bd. 3 (Marx 2003: 413) anmerkt: »Die eingehende Analyse des Kreditwesens und der Instrumente, die es sich schafft (Kreditgeld usw.), liegt außerhalb unseres Plans«, bietet er im Folgenden wesentliche Hinweise zur begrifflichen Klärung, insbesondere in Kap. 29-32; so u. a. Marx (2003: 484): »Aller Zusammenhang mit dem wirklichen Verwertungsprozess des Kapitals geht so (durch die Kapitali- 
neue Techniken seiner Refinanzierung, durch die es seine Kapitalbasis ständig erweitert - siehe dazu die unter (1) genannten Wertpapierkategorien und -volumina. Im Zug dieses Prozesses subsumiert das Finanzkapital alle Sphären der Kapitalakkumulation - durch Hedge Funds, Private Equity Funds, ebenso wie die »normalen «, durch große Kreditkonsortien finanzierten $»$ Mergers \& Acquisitions $\ll^{3}-$ und macht sie zu nachgeordneten Betätigungsfeldern seiner Akkumulation. Das heißt: Kapitalakkumulation existiert nurmehr in der Form der Akkumulation von Finanzkapital. Das gilt auch für Industrieunternehmen. Nicht nur gehören sie als Aktiengesellschaften, deren Eigentumstitel als Wertpapiere an der Börse gehandelt werden, deren Eigentümer sich also in »bloße Geldkapitalisten« (Marx 2003: 452) verwandelt haben, selbst der Sphäre des Finanzkapitals an. Auch ihr industrielles Geschäft ist oft nur noch ein Profit Center neben anderen, zuvorderst den Finanzanlagen ist, an deren Gewinnen es sich messen lass muss (siehe das US-Unternehmen General Electric, dessen Finanzsparte GE Capital in den Jahren vor Ausbruch der Krise über die Hälfte des Konzerngewinns erbrachte, oder - noch drastischer - das Beispiel Porsche, wo die verbuchten Gewinne aus dem Finanzgeschäft im Geschäftsjahr 2007/2008 den Konzernumsatz im industriellen Geschäft übertrafen) ${ }^{4}$ und durch deren Expansion es selbst wesentlich angetrieben wird (siehe etwa die extrem hohe Abhängigkeit des Absatzes großer deutscher Autoproduzenten vom kreditfinanzierten Leasinggeschäft ${ }^{5}$ sowie die nahezu ausschließliche Vorfinanzierung des Absatzes amerikanischer Automobilkonzerne durch Verbraucherkredite). ${ }^{6}$

Ich bezweifele, dass die u. a. von Gerald Epstein (2005) und Tom Palley (2007) popularisierte Finanzialisierungsthese mit dem von ihr unterstellten grundsätzlichen Gegensatz von Produktions- und Finanzsektor, die sich Andreas Nölke und Brigitte Young zu eigen gemacht haben, diesen fundamentalen, dem entwickelten Kapitalismus durchaus »systemisch« inhärenten Subsumtionsprozess adäquat charakterisiert. Wie Nölkes Gegenüberstellung von »Vorteilen« und »Nachteilen« des »finanzialisierten« Kapitalismus (Nölke 2009: 129) zeigt, kommt man auf diesem Weg sehr schnell zu der problematischen normativen Unterscheidung von gutem und schlech-

sierung $>$ jede [r] regelmäßig sich wiederholende[r] Einnahme $<$ bis auf die letzte Spur verloren, und die Vorstellung vom Kapital als einem sich durch sich selbst verwertenden Automaten befestigt sich «.

3 Der enorme Aufschwung dieser Aktivitäten wäre ohne die neuen Refinanzierungstechniken der Banken, also die verschiedenen Formen von Kreditverbriefungen, mit denen Bankkredite mitsamt dem damit verbundenen Kreditrisiko in handelbare Wertpapiere verwandelt werden, nicht denkbar gewesen. Und angesichts ihrer systemwesentlichen Bedeutung ist es auch kaum wahrscheinlich, dass die Regulierer die Kreditverbriefung ernsthaft erschweren werden.

4 »Porsche macht mehr Gewinn als Umsatz«, Handelsblatt 7.11.2008, (http://www.handelsblatt.com/unternehmen/industrie/porsche-macht-mehr-gewinn-als-umsatz\%3B2082709; 5.10.09.).

5 »Die Münchner haben jahrelang mit günstigen Leasing- und Kreditangeboten ihren Absatz angekurbelt «, Frankfurter Allgemeine Zeitung, 4.11.2008, (http://www.fazfinance.net/Aktuell/Wirtschaft-und-Konjunktur/BMW-ruft-groesste-Krise-der-Unternehmensgeschichteaus-1823.html; 5.10.2009.

6 Selbst die ehrwürdige Einrichtung deutscher mittelständischer Familiengesellschaften taugt nicht mehr als Gegenbeweis gegen die Subsumtion des industriellen unter die Akkumulation des Finanzkapitals, siehe die Fälle Schäffler, Schickedanz/Quelle und Adolf Merkle. 
tem Kapitalismus, die man dann auch gleich auf den »guten« rheinischen oder auch den »fordistischen « Kapitalismus einerseits, und den »schlechten « angelsächsischen oder auch »neoliberalen « Kapitalismus andererseits anwendet. Letztlich landet man auf diese Weise bei der unseligen Differenzierung von »gutem«, weil produktivem industriellem und »schlechtem«, weil spekulativem Finanzkapital, wie sie gerade in Deutschland und den anderen Ländern des rheinischen Kapitalismus die gegenwärtige Krisendiskussion bestimmt. Auch Bieling (2009: 112-115) gerät auf diese schiefe Bahn, wenn er den überhand nehmenden »Finanzmarktkapitalismus« zu dem Faktor erklärt, der die Besonderheit der aktuellen Krise ausmacht.

Aus allen vergleichenden Kapitalismusstudien, intertemporalen wie interregionalen (und letztere lassen sich unter dem Aspekt der Ungleichzeitigkeit analytisch letztlich auch in erstere auflösen) lässt sich, hegelianisch (und damit in diesem Punkt auch mit Marx) argumentierend, schlicht eine immer weiter voranschreitende Kapitalisierung des real existierenden Kapitalismus ableiten. Die Subsumtion allen Wirtschaftens unter die Akkumulation des Finanzkapitals ist keine historische Verirrung, sie ist der Logik des Kapitalismus inhärent und als solche grundsätzlich bereits im Verhältnis Ware - Geld angelegt. ${ }^{7}$ Sie ist somit der Grund für die »Systemrelevanz« des Finanzsektors, aufgrund derer die betroffenen Staaten zwar mit erheblichen Haushaltsmitteln die Finanzinstitute retten, gegenüber notleidenden Unternehmen des Industrie- und Nicht-Finanz-Dienstleistungssektors aber wesentlich zurückhaltender mit dem Einsatz öffentlicher Gelder sind.

Die idealistische Idylle einer Kreditwirtschaft im Dienste des redlich schaffenden industriellen Kapitals, auch wenn ihr seit dem Ende von Bretton Woods bis zum heutigen Tage gerade kritische Geister ${ }^{8}$ anhängen, ist im entwickelten Kapitalismus nicht zu haben. Diesen Idealismus als Ziel einer Finanzmarktregulierung zu postulieren, führt daher in die Irre. ${ }^{9}$ Vielmehr hat sich die Banken- und Finanzmarktregulierung gerade darin an der »Systemrelevanz « des Finanzsektors zu orientieren, dass sie seiner Akkumulation nur die nötigsten, für sein eigenes Funktionieren unerlässlichen Beschränkungen auferlegt.

(3) Das Material der Akkumulation des Finanzkapitals sind die von den Zentralbanken emittierten nationalen Währungen. Welche Währungen bevorzugt werden, ist eine Sache der Konkurrenz auf den internationalen Finanzmärkten - und zwar nicht nur der Konkurrenz unter den Finanzinstituten, sondern der Konkurrenz der emittierenden Staaten, für die die internationale Geltung ihrer Währung eine entscheidende ökonomische Grundlage ihrer politischen Macht ist, nicht nur als Ausdruck ihrer internationalen Kreditwürdigkeit und damit -fähigkeit. Da, wie unter (2) ausgeführt, die gelingende nationale Kapitalakkumulation als solche eine Funktion der Akkumulation des Finanzkapitals ist, hat die nationale wirtschaftliche Leistungsfähigkeit ein

7 Marx (2003: 413) verweist im 25. Kapitel des 3. Bandes »Kredit und fiktives Kapital« selbst auf den Abschnitt »Zahlungsmittel« im 3. Kapitel des 1. Bandes (Marx 1988: 148-156).

8 Vgl. Strange (1998) und Altvater (2009).

9 Diese Kritik gilt nicht für Oliver Kessler (2009), der sich statischer, eindimensionaler Erklärungsversuche und Lösungsvorschläge zur Krise bewusst enthält, vielmehr aufzeigt, warum solche Versuche den Gegenstand und ihr Ziel verfehlen. 
entscheidendes Maß in der Nachfrage nach der eigenen Währung als Material für dessen Akkumulation. Deshalb ist auch die Krisenbewältigung für die Staaten nicht nur eine Frage der innenpolitischen Stabilität, sondern vor allem auch eine Frage der nationalen Selbstbehauptung im internationalen Wettbewerb der Staaten. Und das führt mitten in die Frage, welche Auswirkungen die Krise auf die internationale Ordnung hat. Denn diese ist ja nun seit jeher eine Konkurrenz-Ordnung, mit der Betonung einmal mehr auf Konkurrenz, einmal mehr auf Ordnung, je nachdem wie weit ein internationales Regelsystem im Rahmen einer Hegemonialordnung an die Stelle rein machtbasierter Konkurrenz tritt und eine weltweite Leitwährung als Währung einer Hegemonialmacht das Maß aller Dinge für den wirtschaftlichen Erfolg der einzelnen Nationen ist.

Von daher ist es sinnvoll, die Frage nach den Auswirkungen der Krise auf die internationale Ordnung dahingehend umzuformulieren, wie sich diese in der Konkurrenz der Staaten niederschlägt.

Das ist eine eminent politisch relevante Frage. Auf der einen Seite steht eine wachsende Zahl von Verlierer-Staaten, von denen manche angesichts fehlender innerer Stabilität nahe an den Status eines failing state heranrücken - als ein Beispiel mag hier die Ukraine gelten. Auf der anderen Seite betont noch jeder Regierungschef jeder maßgeblichen Wirtschaftsmacht die Absicht, sein Land nicht nur gestärkt, sondern stärker als die anderen aus der Krise zu führen. Nicht allen wird das gelingen.

Man kann sagen: gerade weil derzeit noch so etwas wie eine internationale Wirtschafts- und Finanzordnung, also ein intaktes Regelsystem für die internationale Konkurrenz der Staaten existiert, nehmen die Anstrengungen der Staaten zu, innerhalb oder auch hart am Rande dieser Regeln ihre Konkurrenzposition als Standort im internationalen Handel und Kapitalverkehr zu verbessern sowie ihren Einfluss auf andere, schwächere Staaten auszubauen. Das Streben nach dem relative gain im Rahmen des gemeinsam erlittenen krisenbedingten absoluten Verlustes wird angesichts der Höhe dieses Verlustes um so heftiger, wie der wachsende Wirtschaftsnationalismus und der zunehmende Protektionismus bei der Krisenbewältigung zeigen.

\section{Internationale Regulierungskonkurrenz}

Gleichwohl hat noch keine maßgebliche Wirtschaftsnation dem multilateralen Handelsregime der WTO oder den beiden Bretton Woods-Institutionen IWF und Weltbank eine Absage erteilt; darin zumindest beweist sich die Stabilität der gegenwärtigen internationalen Wirtschaftsordnung. Vielmehr wird die krisenbedingt verschärfte Konkurrenz der Staaten nicht nur mit unilateralen marktbeeinflussenden Maßnahmen ausgetragen, sondern auch auf der Ebene der künftigen Regulierung der internationalen Finanzmärkte selbst: innerhalb der EU zwischen kontinentaleuropäischen und angelsächsisch geprägten Ansätzen und Interessen, im transatlantischen Kontext zwischen Europäern und den USA, und darüber hinaus nun auch zwischen dem »Westen« und den großen neuen Wirtschaftsmächten, allen voran China - mit der G20 und dem IWF als den derzeit wichtigsten Foren. Niemand, erst recht nicht die USA, will eine 
internationale Regulierung akzeptieren, die die Wettbewerbsposition des eigenen Finanzsektors zu Lasten derjenigen anderer Staaten schwächt. Zwangsläufiges Resultat dieser scharfen »regulativen Konkurrenz« wird ein Minimalkonsens sein, der der ökonomischen Konkurrenz der Staaten nur wenig enger gezogene Schranken setzt als bislang. Die Tobinsteuer als weltweite Finanzmarktsteuer wird wohl kaum dazu gehören.

Für eine produktive Analyse dieser Situation ist es also hilfreich, das hier kurz beschriebene Verhältnis von Konkurrenz und Ordnung als dialektisches im Auge zu behalten. Das sei kurz an Bieling (2009) erläutert. Bielings Aufsatz stellt die aktuelle Krise zunächst in das historische Kontinuum kapitalistischer Kreditkrisen, wie es Charles Kindleberger und Hyman Minsky analysierten, um dann im zweiten Teil ihre Besonderheiten herauszuarbeiten. Im ersten Teil kann ich ihm gut folgen, im zweiten nicht mehr. Auch hier ist eine dialektische Betrachtungsweise hilfreich: Es genügt ja nicht, zu konstatieren, dass in den ersten 25 Jahren nach der Konferenz von Bretton Woods die Welt unter der regelsetzenden Hegemonialmacht USA noch in Ordnung war, dann aber wegen der zunehmenden Finanzmarktliberalisierung in den 1970er und vor allem 1980er Jahren aus den Fugen geriet. Denn die USA selbst waren der maßgebliche Akteur dieses regimeändernden Liberalisierungsprozesses und sie hatten dafür gute Gründe. Die von der Hegemonialmacht aufgestellten Regeln der bisherigen Ordnung behinderten diese immer mehr selbst in ihrer sich verschärfenden Konkurrenz mit den aufsteigenden Wirtschaftsmächten Westeuropas und Japan, bei gleichzeitig wachsendem Aufwand für die politisch-militärische Absicherung ihrer Hegemonieordnung, wie er sich aus dem Vietnamkrieg, vor allem aber auch aus dem Rüstungswettlauf mit der UdSSR ergab. Die Liberalisierung der Währungsmärkte nach dem Ende von Bretton Woods stärkte die Nachfrage nach US-Dollar (wie die Erfolgsgeschichte - aus Sicht der USA - des Petrodollar-Recycling ab 1974 zeigt) und verschaffte dem US-Finanzsektor wieder einen Wettbewerbsvorteil, der durch die Finanzmarktderegulierung bei gleichzeitiger monetaristischer Neuausrichtung der Zentralbankpolitik unter Reagan als Präsident und Paul Volcker als Fed-Vorsitzendem noch ausgebaut wurde. Auf diese Weise gelang es einerseits, den Fortbestand amerikanischer Hegemonialmacht auch nach dem Ende des Ost-West-Gegensatzes ökonomisch-monetär abzusichern und die allfälligen Finanzkrisen andernorts stattfinden zu lassen - die Globalisierung der 1990er Jahre belegt dies eindrucksvoll, einschließlich ihrer Krisen (1994 Mexiko; 1997/98 Asien, Russland; 2001 Argentinien). Andererseits wurde damit auch eine lebhafte internationale Deregelierungskonkurrenz hinsichtlich der Finanzmärkte angefeuert, die den Finanzmarktakteuren neue Möglichkeiten der Kapitalakkumulation eröffnete - mit den bekannten Folgen.

Wenn dieser Liberalisierungswettlauf nun unter dem Eindruck der gegenwärtigen Krise zu einem Ende kommt, hört damit gewiss nicht die Konkurrenz um die Finanzmarktregulierung auf. Im Gegenteil: In dem Maße, wie die regelsetzende Hegemonialmacht der USA erodiert, wird sich die Konkurrenz um Art und Ausmaß künftiger internationaler Finanzmarktregulierung - immer auf der Grundlage eines gemeinsamen Interesses, dass es einer solchen Regulierung bedarf - eher verschärfen, wie an Verlauf und Ausgang der G20-Gipfelkonferenzen des Jahres, von London und Pitts- 
burgh, abzulesen ist. Populäre Statements à la »das angelsächsische Regulierungsmodell ist gescheitert« sollten nicht als Zeichen einer Trendwende weg vom »Finanzmarktkapitalismus« fehlinterpretiert sondern als durchaus interessengeleitete Argumente in der gegenwärtigen Konkurrenz um die künftige Finanzmarktregulierung gesehen werden.

Sich eindimensional auf die liberal-institutionalistische Seite zu stellen und aus der Krise die Notwendigkeit von mehr Global Governance für die Finanzmärkte abzuleiten, ${ }^{10}$ mag zwar ein nachvollziehbarer erster Reflex aus der (Fehl-)Diagnose $»$ Regulierungsversagen« als Ursache der Krise sein, erscheint aber ebenso wenig fruchtbar für ein besseres Verständnis der in der Form internationaler Verhandlungsprozesse tatsächlich ausgetragenen zwischenstaatlichen Standortkonkurrenz im Gefolge der Finanzkrise wie eine rein realistische Betrachtung, die sich auf eine Analyse der krisenbedingten Machtverschiebungen zwischen den Staaten beschränkt. Ergiebiger dürfte es sein, in den gegenwärtigen Auseinandersetzungen diese Dialektik von Konkurrenz und Ordnung herauszuarbeiten, die mit der möglichen Ablösung des seit dem Zweiten Weltkrieg bestehenden regulativen Hegemonialsystems die internationale Finanzordnung mittelfristig auf eine neue, eher multipolar strukturierte Ebene führen könnte.

Es ist noch zu früh für fundierte Aussagen, wie eine solche multipolare, also nichthegemoniale Ordnung, ein solches »leaderless currency system« (Cohen 2009) aussehen kann. Es ist aber nicht unwahrscheinlich, dass entgegen manchen derzeitigen Erwartungen das Ausmaß internationaler Regulierung gegenüber konkurrierenden nationalen Regulierungen eher zurückgehen wird. Und die maßgeblichen Institutionen der internationalen Wirtschafts- und Finanzordnung dürften sich von Instanzen hegemonialer Ordnungssetzung zu den wichtigsten Foren der Austragung der internationalen ordnungspolitischen Konkurrenz entwickeln.

\section{Die G20 und die Neuverteilung der Gewichte in der internationalen Wirtschafts- und Finanzordnung}

Dies sei abschließend am Beispiel der G20 erläutert. Die G20, die erst seit dem vollen Ausbruch der Finanzkrise im Herbst 2008 auf Gipfelebene zusammentritt, am 25./26. September 2009 zum dritten Mal innerhalb von 12 Monaten, ist in kurzer Zeit zum maßgeblichen institutionellen Ort für das Bemühen um eine neue Ordnung für die Weltwirtschaft geworden und hat die G7/8 faktisch in die Bedeutungslosigkeit gedrängt (Frankel 2009: 2, 16), sodass deren Auflösung für 2011 erwartet wird, wenn Frankreich die Präsidentschaft sowohl von G20 wie von G7/8 innehat. ${ }^{11}$ Diese Entwicklung mag befördert worden sein durch die historische Koinzidenz, dass die letzten beiden G7/G8-Präsidentschaften (Japan 2008, Italien 2009) als besonders

10 So etwa Schirm (2009), Angeloni (2009: 4-7), Dieter (2009: 3-8).

11 » Crisis leaves G7 on its last legs «, Financial Times, 2.10.2009, (http://www.ft.com/cms/ s/b805dd18-af8a-11de-ba1c-00144feabdc0, dwp uuid=abb716b0-2f7a-11-

da-8b51-00000e2511c8,print=yes.html; 5.10.2009). 
schwach, im Sinne geringer Gestaltungs- und Durchsetzungsmacht gegenüber den anderen Mitgliedern der Gruppe, bezeichnet werden können. Entscheidender Faktor aber ist die Finanzkrise, da sie gerade von den führenden westlichen Industrieländern der G7 (vielleicht mit Ausnahme Japans) ausging und so deren ohnehin schon lädierten Ordnungsanspruch delegitimierte.

Dass die Rolle des maßgeblichen globalen wirtschafts- und finanzpolitischen Koordinationsforums nun von den G7 auf die G20 übergegangen ist, stellt einen bemerkenswerten Wandel dar, wird dadurch doch auf institutioneller Ebene der Abschied des »Westens« von seiner hegemonialen Gestaltungsrolle für die Weltwirtschaft eingeleitet. ${ }^{12}$ In der G20 sitzen nun die für die Zukunft der internationalen Finanz- und Währungsordnung entscheidenden beiden Akteure, die USA und China, an einem Tisch. Ihr dialektisches Verhältnis von Rivalität und Interdependenz dürfte diese Zukunft stärker prägen als die innerwestliche Konkurrenz zwischen EU und USA, zwischen Euro und Dollar.

Die Widersprüchlichkeit dieses Verhältnisses zeigt sich schon daran, dass einerseits die enge ökonomische Symbiose der beiden Wirtschaftsmächte, die Abhängigkeit Chinas vom amerikanischen Markt und die Abhängigkeit der USA von der Finanzierung ihres Leistungsbilanzdefizits durch China einer der wichtigsten Faktoren gewesen sein dürfte, die zu der krisenträchtigen Überakkumulation des amerikanischen Finanzsektors beitrugen, dass sich andererseits jetzt die größten Hoffnungen für eine Überwindung der Krise eben auf China richten, das von der Krise nur geringer betroffen und sich am schnellsten zu erholen scheint.

Das ökonomische Verhältnis der USA zu China spielt auch eine besondere Rolle für die Erklärung der krisenträchtigen Entwicklung der US-Wirtschaft seit 2001 selbst, wie im folgenden kurz erläutert werden soll.

Die von Young (2009) analysierte privatkeynesianische Nachfrageexpansion, wurde - das wird auch bei Young implizit deutlich - durch den radikalen steuer- und fiskalpolitischen Kurswechsel angestoßen, den George W. Bush unter dem Eindruck von 9/11 im Kongress durchgesetzt hat. Zuvor, in den letzten beiden Jahren der Präsidentschaft Clinton, hatte es ja erhebliche Budgetüberschüsse gegeben. Und das fortbestehende Leistungsbilanzdefizit war bis dahin eher Konsequenz der hohen Zuflüsse an privatem Kapital - vor allem Direktinvestitionen und Portfolioinvestitionen am amerikanischen Aktienmarkt. Es lässt sich daher gut begründen, dass der »privatisierte Keynesianismus « seinen entscheidenden Schub erst unter Bush erhielt. Dieser hat sich dabei sicher auch auf die von Young angesprochenen bei seiner Wählerbasis populären ideologischen Argumente à la »ownership society « gestützt, die von zahlreichen radikal antietatistischen advocacy groups propagiert wurden. Mastermind dieser Politik war aber sein Vizepräsident Dick Cheney, der schon unter Reagan eher unideologisch eine rücksichtslose Defizitexpansion (»deficits don't matter«) zur Finanzierung der außen- und sicherheitspolitischen Aufwendungen vertreten hatte und

12 Dieser Abschied könnte mit der für Anfang 2011 angekündigten deutlichen Neuverteilung der Kapital- und Stimmrechtsquoten im IWF weitergehen. Zu diesem Thema eingehender: van Scherpenberg (2009). 
dies als Politik von »guns and butter« zur innenpolitischen Legitimation des »Global War on Terror « auch diesmal vertrat. ${ }^{13}$ Geldpolitisch konsequent flankiert wurde diese Politik durch einen anderen Veteran der Reagan-Ära, Alan Greenspan.

Erst im Zuge dieser Politik kam es zu einer fundamentalen Veränderung in der Zusammensetzung der Kapitalimporte der USA, also der Finanzierung ihres Leistungsbilanzdefizits, die bei Young unter den Tisch fällt. ${ }^{14}$ Die Direktinvestitionen fielen drastisch, von $33 \%$ der Kapitalimporte im Durchschnitt der Jahre 1998-2001 auf $10 \%$ durchschnittlich in 2003-2007. Dafür nahmen die Kapitalzuflüsse von ausländischen offiziellen Stellen (in der Regel Zentralbanken) für dieselben Zeiträume von $2 \%(1998-2001)$ auf $25 \%(2003-2007) z^{15}$

Es passt zu diesen Angaben, dass Chinas Leistungsbilanz bis etwa 2001 insgesamt ausgeglichen war und erst ab 2002 allmählich (70 Mrd. Dollar), dann aber mit dem wachsenden Importsog aus den USA einen immer schneller wachsenden Überschuss verzeichnete. Dieser wurde in den folgenden Jahren nicht nur in US-Treasuries, sondern zu einem wachsenden Anteil in Schuldverschreibungen quasi-staatlicher agencies, insbesondere Fannie Mae und Freddie Mac, investiert. Der doppelte Effekt des das Zinsniveau niedrig haltenden hohen chinesischen Dollarrecyclings und des Drucks auf das Verbraucherpreisniveau in den USA durch die billigen Importe aus China sorgte dafür, dass die Fed keinen Grund zum geldpolitischen Eingreifen sah und die Regierung Bush sich weiter im Glauben wiegen konnte, die US-Wirtschaft werde durch überdurchschnittliches Wachstum mittelfristig schon irgendwie ihre Defiziten überwinden.

Die seltsame Symbiose zwischen USA und China, bisweilen auch durch den Ausdruck »Chimerica« illustriert (Ferguson/Schularick 2007), zog das Forschungsinteresse vieler Ökonomen an. Viele sahen hier, an der außenwirtschaftlichen Flanke, das Einfallstor für eine abrupte Anpassungskrise in den USA. Andere betonten, dass diese Situation sich verstärkender bilateraler Ungleichgewichte so lange funktionieren werde, wie das »exorbitant privilege« der USA durch deren impliziten politischen Konsens mit China - von Michael Dooley et al. (2003) als »Bretton Woods II« beschrieben - fortbesteht (Mann/Plück 2006).

Es ist zwar nicht ohne Ironie, dass dieser Konsens immer noch existiert, während die Krise in den USA von innen ausgebrochen ist. Doch aus ökonomischer Sicht ist es nicht verwunderlich, denn beide Seiten haben derzeit praktisch keine anderen Optionen. Die Warnung vor einer Erpressbarkeit der USA durch Chinas hohe Dollarreserven und treasury investments entbehrte von jeher jeder ökonomischen Grundlage.

Dennoch hat sich im Verhältnis zwischen beiden Mächten politisch seit Ausbruch der Krise etwas geändert. Nicht nur hat China im August 2008, noch vor dem Zu-

13 Vgl. hierzu und zum folgenden van Scherpenberg (2004: 24-28).

14 Vgl. Young (2009:151f).

15 Noch ausgeprägter fällt der Vergleich der beiden Zeiträume bei der Betrachtung des Saldos der Direktinvestitionen (ausländische DI in den USA minus amerikanische DI im Ausland) aus. Als Anteil am Saldo der Kapitalbilanz (Überschuss der Kapitalimporte über die -exporte) fielen sie von $18 \%$ (1998-2001) auf -9\% (2003-2007), also ein Nettoabfluss bei DI, Bureau of Economic Analysis, U.S. International Transactions; (www.bea.gov/ international; 12.10.2009). 
sammenbruch der Lehman Bank, durch die Drohung, seine Bestände an Schuldverschreibungen der großen US-Hypothekenbanken Fannie Mae und Freddie Mac zu verkaufen, die US-Regierung faktisch genötigt, die beiden Banken formell zu verstaatlichen. Seit Ende 2008 hat die chinesische Regierung auch immer deutlicher erkennen lassen, dass sie nicht nur mit ihrem »Co-Dependence«-Verhältnis zu den USA (Mann 2004) unzufrieden ist, sondern die Rolle des Dollar als Welt-Leit- und Reservewährung insgesamt in Frage zu stellen gedenkt. Zwar sind ihre Möglichkeiten, ihre Währungsreserven zu Lasten des Dollar stärker zu diversifizieren, begrenzt, wie Richard Cooper (2009) zeigt. Doch schließt Cooper zu kurz, wenn er die Rolle des Dollar deshalb noch auf längere Sicht unbeeinträchtigt sieht. Sowohl unilateral wie auf multilateraler Ebene verfolgt China gegenwärtig Strategien, die die weltwirtschaftliche Leit- und Ordnungsfunktion der USA nicht unbeeinträchtigt lassen werden.

Unilateral wird nicht ohne Erfolg der Aufbau eigener Strukturen außerhalb der multilateralen Handels- und Finanzinstitutionen betrieben. So werden in wachsendem Maße Dollarreserven für Auslandsinvestitionen vor allem in Rohstoffressourcen eingesetzt. Zudem sind seit Ende 2008 mit einer Reihe von Handelspartnern swap-Abkommen geschlossen worden, die die Verwendung des Renminbi als Transaktionswährung im bilateralen Handel vorsehen. ${ }^{16}$ Regional verstärkt China seine Bemühungen um eine asiatische Wirtschaftsintegration im ASEAN plus 3-Kontext. Zu gute kommt China dabei auch der soft-power-Effekt: Nicht nur hat die durch die Finanzkrisen der 1990er Jahre außerhalb des Westens ohnehin bereits angeschlagene Reputation des amerikanischen Wirtschaftsmodells und der USA als weltwirtschaftliche Ordnungsmacht durch die gegenwärtige Finanzkrise vollends massiv Schaden genommen. Die Tatsache, dass China durch die Krise vergleichsweise geringer getroffen wurde und sie offenbar als erste große Wirtschaftsmacht zu überwinden im Begriff ist, hat in Asien, Afrika und Lateinamerika zu einem enormen Prestigegewinn für China zu Lasten der USA geführt.

China kann mithin in der neuen G20 mit wesentlich gestärkter Position als nahezu ebenbürtiger Gegenspieler der USA auftreten. Die chinesische Initiative von März 2009 (Zhou Xiaochuan 2009), den US-Dollar als globale Leit- und Reservewährung durch die IWF-Sonderziehungsrechte (SZR) zu ersetzen, ist zwar als durchaus langfristig gemeinter Vorstoß zu verstehen (siehe dazu aber Williamson 2009). Immerhin aber konnte China auf den Konferenzen von April 2009 (G20-Gipfelkonferenz in London und Frühjahrskonferenz von IWF und Weltbank) erreichen, dass im Rahmen einer von den USA vorgeschlagenen Verdreifachung des IWF-Kapitals auf $750 \mathrm{Mrd}$. Dollar die Emission von SZR auf über $300 \mathrm{Mrd}$. Dollar mehr als verzehnfacht wurde.

China agiert inzwischen in den G20 als faktische Führungsmacht der dort präsenten großen emerging market-Staaten (neben China Argentinien, Brasilien, Indien und Südafrika). Und es ist durchaus fraglich, ob sich dieser Gruppe ein vereinter Westen entgegenstellen wird oder ob die USA und die europäischen G20-Staaten bei der

16 Solche Abkommen sind bislang abgeschlossen worden mit Hongkong, Malaysia, Indonesien, Südkorea, Belarus, Argentinien, Brasilien. Russland verhandelt ebenfalls über den Abschluss eines swap-Abkommens mit China. 
künftigen Gestaltung der internationalen Wirtschaftsordnung in wechselnden Allianzen agieren werden, um ihre eigene Verhandlungsposition zu stärken.

Die Formeln, die auf der G-20-Gipfelkonferenz Konsens fanden (G-20 2009), ähneln zwar manchen Formulierungen früherer G7-Kommuniques; jedoch fehlen die macht- und ordnungspolitischen Voraussetzungen dafür, dass dieser Formelkonsens die gleiche praktische Bedeutung entfaltet wie etwa die hegemonial geprägten Vereinbarungen der New Yorker G7-Konferenz von1985, der »Plaza Accord «.

Es muss zunächst offen bleiben, wie westlich die aus der Krise hervorgehende internationale Wirtschafts- und Finanzordnung in ihren Grundsätzen sein wird. Dass sie kein Instrument westlicher Gestaltungshegemonie mehr sein, sondern durch eine intensivierte Konkurrenz der großen Wirtschaftsmächte um die Akkumulationsbedingungen des von ihrem Boden aus operierenden Kapitals und um die Tauglichkeit ihrer nationalen Währungen als Material dieser Akkumulation geprägt wird, ist jedoch bereits deutlich zu erkennen.

\section{Literatur:}

Altvater, Elmar 2009: Die Finanzkrise ist eine Systemkrise des Kapitalismus, in: Leviathan 37: 2, 197-210.

Angeloni, Ignazio 2009: The Pittsburgh G20 Checklist (Bruegel Policy Contribution 09), in: http://www.bruegel.org/nc/publications/show/publication/the-pittsburgh-g20-checklist.html; 18.10.09.

Bieling, Hans-Jürgen 2009: Wenn der Schneeball ins Rollen kommt. Überlegungen zur Dynamik und zum Charakter der Subprime-Krise, in: Zeitschrift für Internationale Beziehungen 16: $1,107-122$

Cohen, Benjamin J. 2009: Towards a Leaderless Currency System, in: Helleiner, Eric/Kirshner, Jonathan (Hrsg.), The Future of the Dollar, Ithaca, NY, 142-163.

Cooper, Richard N. 2009: The Future of the Dollar (Peterson Institute for International Economics Policy Brief, PB 09), in: www.iie.com/publications/interstitial.cfm?ResearchID $=1290 ; 5.10 .2009$.

Dieter, Heribert 2009: Aufbruch oder kosmetische Reformen in Pittsburgh? Die Chancen für eine umfassende Neuordnung der internationalen Finanzmärkte (SWP-Aktuell 53), in: http://www.swp-berlin.org/common/get_document.php?asset id=6336; 18.10.09.

Dooley, Michael P./Folkerts-Landau, David/Garber, Peter 2004: An Essay on The Revived Bretton Woods System, in: International Journal of Finance and Economics 9: 4, 307-313.

Epstein, Gerald (Hrsg.) 2005: Financialization and the World Economy, Cheltenham.

Ferguson, Niall/Schularick, Moritz 2007: Chimerica and Global Asset Markets, in: http:// www.jfki.fu-berlin.de/faculty/economics/persons/schularick/chimerica.pdf; 18.10.09.

Frankel, Jeffrey 2009: On Global Currencies (Harvard Kennedy School, Faculty Research Papers Working Series, RWP 09-026), in: http://web.hks.harvard.edu/publications/workingpapers; 5.10.2009.

G-20 2009: Leaders' Statement: The Pittsburgh Summit September 24-25, 2009, in: http:// www.pittsburghsummit.gov/mediacenter/129639.htm; 28.9.2009.

Kessler, Oliver 2009: Die Subprime-Krise und die Frage nach der Finanzmarktstabilität, in: Zeitschrift für Internationale Beziehungen 16: 1, 161-176.

Krugman, Paul 2009: How Did Economists Get It So Wrong? in: The New York Times Magazine, 6.9.2009 (http://www.nytimes.com/2009/09/06/magazine/06Economic-t.html; $5.10 .2009)$. 
Mann, Catherine/Plück, Katharina 2006: The United States as Net Debtor: How Much Longer the »Exorbitant Privilege«? in: van Scherpenberg, Jens/Plück, Katharina (Hrsg.): Sharing the Growing Economic Burden of World Order, Berlin, 33-47.

Mann, Catherine 2004: Managing Exchange Rates: Achievement of Global Re-balancing or Evidence of Global Co-dependency? in: Business Economics 39: 3, 20-29.

Marx, Karl 1988: Das Kapital, 1. Bd., (Marx-Engels Werke Bd. 23), 17. Aufl., Berlin.

Marx, Karl 2003: Das Kapital, 3. Bd., (Marx-Engels Werke Bd. 25), 18. Aufl., Berlin.

Nölke, Andreas 2009: Finanzkrise, Finanzialisierung und Vergleichende Kapitalismusforschung, in: Zeitschrift für Internationale Beziehungen 16: 1, 123-140.

Palley, Thomas I. 2007: Financialization: What it is and Why it Matters (University of Masachusetts Working Paper 153), in: http://www.peri.umass.edu/fileadmin/pdf/working_papers/working papers_151-200/WP153.pdf; 5.10.2009.

Schirm, Stefan 2009: Koordinierte Weltwirtschaft? Neue Regeln für effizientere und legitimere Märkte, in: Zeitschrift für Internationale Beziehungen 16: 2, 311-324.

Strange, Susan 1998: Casino Capitalism, Oxford.

van Scherpenberg, Jens 2004: Der geborgte Aufschwung. Die wirtschaftspolitische Bilanz der Regierung Bush 2001 - 2004 (SWP-Studie S-40), in: http://www.swp-berlin.org/common/ get document.php?asset id=1689; 5.10.2009.

van Scherpenberg, Jens 2009: Assault on the Last Fortress of Western Order? International Economic and Financial Institutions in the Financial Crisis (Papier vorgestellt auf der Konferenz Uses of the West, Bad Homburg 8.10.09), unv. Ms.

Williamson, John 2009: Why SDRs Could Rival the Dollar (Peterson Institute for International Economics Policy Brief 09-20), in: http://www.piie.com/publications/pb/pb09-20.pdf; 5.10.09.

Young, Brigitte 2009: Vom staatlichen zum privatisierten Keynesianismus. Der globale makroökonomische Kontext der Finanzkrise und der Privatverschuldung, in: Zeitschrift für Internationale Beziehungen 16: 1, 141-160.

Zhou Xiaochuan 2009: Reform of the International Monetary System (People's Bank of China), in: http://www.pbc.gov.cn/english/detail.asp?col=6500\&id=178; 5.10.2009. 